\title{
Nonlocal Mean Filtering Algorithm for Low Contrast Images and its Application
}

\author{
Tianyi Guan* \\ Sendelta International Academy, 518000 \\ *Corresponding Author.
}

\begin{abstract}
High background noise, cracks, fuzzy boundaries image containing the chromatism, etc are the common problems faced in the low contrast image recognition, this paper takes the core fracture identification of two-dimensional section as an example, and highlight the point, simplify the problem, this paper only considering the three dimensional images of two-dimensional cross section along the direction perpendicular to the core shaft, focusing on the identification of disc core cracks within the dark grey. Each voxel based on 3D digital images corresponds to a gray value. The smaller the value, the blacker the corresponding voxel will be. The larger the value, the whiter the corresponding voxel is. By fine-tuning the background color difference, filtering and denoising, marking the non-crack area, secondary denoising by graphics method and other algorithm methods, the identification efficiency of the crack area in the two-dimensional cross-section diagram of the core column is effectively improved. This method can also be used as a solution to other problems in similar scenes.
\end{abstract}

Keywords: High noise, the crack boundary is fuzzy, fine tuning background chromatic aberration, filtering and denoising, mark non-fractured areas

\section{Introduction}

In the research process of image segmentation ${ }^{[1]}$, image recognition ${ }^{[2]}$ is a very important work, mainly based on threshold segmentation method, region based segmentation method and edge based segmentation method for image threshold segmentation ${ }^{[3]}$, region segmentation ${ }^{[4]}$ and edge segmentation ${ }^{[5]}$ methods for a comprehensive study and evaluation of cutting methods ${ }^{[6]}$. A relatively mature and reliable method has been developed for the recognition of binary gray-scale images ${ }^{[7]}$, which can quickly recognize the gray value of images when the gray value error is less than 10 . However, the image recognition under low contrast ${ }^{[8]}$ still needs to be further improved, mainly because the noise of the image is greater than that of the conventional binary grayscale image, and the image after noise reduction needs to be visually enhanced again, which directly increases the time cost and the result is not ideal.

With the development of time, there are more and more methods to solve this problem, especially the rapid screening of binary images. However, it is still a research difficulty to perform visual enhancement and reduce time cost on the basis of existing rapid screening. This paper takes the fracture identification of core CT scan image ${ }^{[9-10]}$ as an example, and discusses the image changes brought by each process from four aspects, namely fine tuning background color difference, filtering denoising, marking non-fractured region and graph-based secondary denoising, to solve this problem.

Each voxel in a THREE-DIMENSIONAL digital image (or data body) (analogous to the pixels in a twodimensional digital image) corresponds to a gray value (for example, an integer between 0 and 255 for an 8-bit data body), the smaller the value, the darker the voxel, and the larger the white. The gray value of air is close to 0 , which is black. Hole seam is about 60 70, dark gray; the highest gray value of the rock is close to 90, which is light gray. In theory, it is very easy to distinguish rocks from cracks by setting only segmented thresholds. But on the other hand, due to the scanning X-ray CT unit intensity is not stable, in the image should be the place of the single grey value, there will be a large number of blinks on random noise, air area of grey value, it is not so close to

ISSN: 0010-8189

(C) CONVERTER 2020

www.converter-magazine.info 
zero, but within the range of $[0,40]$, the corresponding hole seam area of grey value falls within the range of [50, 90] and rock area of grey value is located in the [70, 110]. The grayscale of the hole slot area and the rock area will overlap, which means high noise and fuzzy boundary between regions. Rays in the process of penetrating the core, on the other hand, there are a lot of energy attenuation, the deep into the core, the energy attenuation, and the radiation energy (the core) as to the sample density is positive correlation, so the image overall reflect core internal partial black, the characteristics of the white edge, this kind of background color, and the high noise, combined fuzzy boundary effect, make the rock and fracture line between hidden in the mists, difficult to catch. Moreover, we also found that when there are redundant low-frequency waves in the image, the difference of light and shade on the background often appears, so a clever high-pass filter is needed to fill the difference of light and shade on the background without affecting the image quality. In order to solve various problems of image processing, the axial two-dimensional cross-sectional image of cylinder core containing cracks is studied from the perspective of image processing, and a general technique to identify the crack area in the cross-sectional image is analyzed and proposed.

In order to highlight the key points and simplify the problem, this study only considers the two-dimensional section along the direction perpendicular to the core axis in the THREE-DIMENSIONAL image and focuses on identifying the dark gray fractures inside the disk-like core. The $2 \mathrm{~d}$ section of the core has included all the above features of color difference, image high noise and fuzzy boundary (Figure 1), which solves the problem of fracture identification of $2 \mathrm{~d}$ section of the core. The fracture identification of $3 \mathrm{~d}$ image of the core can be extrapolated similarly.

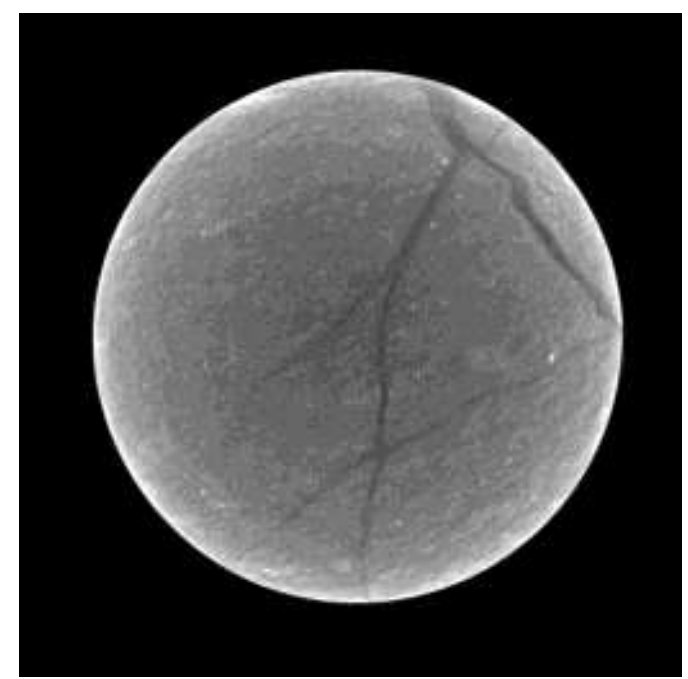

Figure 1: Two-dimensional cross-section of cylinder core of fractured reservoir obtained by micron CT scan

\section{Fracture Identification of Two-Dimensional Core Section}

There are two common methods for image recognition: one is to mark different thresholds in different regions; the other is to obtain the boundary of the region to be recognized by edge detection. Since the edge of the target image in this study is too fuzzy, the difficulty of edge detection may be much higher than the threshold marker, so we choose the former as the guiding method. To identify cracks, they need to be marked. Visually, the contrast between the fractured region and the non-fractured region should be improved first. The most direct method is image filtering and denoising. However, filtering operation will cause great changes to the original image, and various image filters need to be used carefully before the original image is processed in detail. Fine-tuning the background color of the image here is a scalpel type of detail processing. Under this guiding principle, the processing of the target image consists of the following four steps: fine tuning background color difference, filtering denoising, marking non-fractured region, and secondary denoising by graphic method.

\subsection{Fine tune background chromatic aberration}

ISSN: 0010-8189

(C) CONVERTER 2020 
When there are redundant low-frequency waves in the image, the difference between light and dark is often shown in the background, so an ingenious high-pass filter needs to be designed to fill the difference between light and dark in the background without affecting the image quality. In most cases, fast Fourier transform ${ }^{[11]}$ can be performed on the original image to selectively remove the low-frequency components, and then return to the original space through inverse transform, so as to remove the background color difference caused by lowfrequency waves. The problem is that the number of low-frequency waves removed in this method is subjective, and when more low-frequency components are removed, the processed image background will have an unnatural checkerboard mode ${ }^{[12]}$. The background of our original image happens to be in multiple low-frequency bands and is not suitable for processing in this way. Considering the characteristics of the disk-shaped core in the original image, and the background color difference of the original image is distributed annular with the center of the disk circle; Based on this geometric feature, we designed the following Algorithm for background chromatic aberration adjustment:

\footnotetext{
Algorithm 1

Step 1: Otsu algorithm ${ }^{[13]}$ was used to divide the original image into two categories: inside the disk (core) and outside the disk (air). The outside of the disk was assigned to 255 to calculate the radius $\mathrm{R}$ of the disk and the position of the center of the circle;

Step 2: For the circle with radius $0 \leq r<R$, calculate the average gray value $v(r)$ of peripheral pixel points;

Step 3: The minimum average gray value $v_{0}$ of all circles is obtained;

Step 4: For circle of $0 \leq r<R$, update the circumference of a circle pixel gray value $v(x, y):=\frac{v(x, y) v_{0}}{v(r)}$.
}

\subsection{Filtering denoising}

After removing the background chromatic aberration from the original image, filter processing can be performed. The filtering here is mainly to eliminate the high frequency noise in the image. The most common filter in this class is the Gaussian low pass filter which belongs to the mean filter class. Usually, after the Gaussian low-pass filtering, the grainy high-frequency noise in the image is eliminated, but the boundary between different regions in the image also becomes fuzzy. This is because the boundary information is also the high frequency information in the image, but the Gaussian low-pass filter does not differentiate it from the high frequency noise. We need to solve the main contradiction, that is, to eliminate the high frequency noise while keeping the boundary information in the image as much as possible. Therefore, Algorithm 2, an improved version of Gaussian low-pass filter, is chosen: non-local mean filter ${ }^{[14]}$.

Algorithm 2
Step 1: Set the window length of Gaussian filter as $L$, and for the operation pixel $X$, take a search
window with its center and $L$ as the side length, and two similar Windows with the center to be
determined and $l$ as the side length.
Step 2: Place the center of one of the similar Windows in pixel $X$, traverse each pixel $Y$ of the search
window, and take $Y$ as the center of another similar window. In the traversal process, the gray value
distance ${ }^{[9]} d$ between the two similar Windows is calculated, a quasi-standard deviation parameter h is
set, and the kernel function of the gaussian low pass filter, $e^{-\frac{|X-Y|^{2}}{L^{2}}}$ is modified to $e^{-\frac{|X-Y|^{2}}{L^{2}}-\frac{d^{2}}{h^{2}}}$
Step 3: Improved Gauss low pass filtering is performed for each pixel $X$.

Different from the Gaussian low-pass filter, which has only one parameter $L$, the non-local mean filter also has two parameters, $L$ and $H$, which determine the size of similar window of gray value and the intensity distribution in the filter respectively. The significance of introducing the similar window is that the boundary information in the image is different from the pure local noise, and they are usually similar to the boundary of the surrounding area. If the weight of pixels with non-local similarity is added to the Gaussian low-pass filter, more boundary information can be retained while local noise is filtered. Subjectively, the larger $l$ value is, the better the effect will be. However, if 1 value is too large, the calculation amount of gray value distance $d$ will be greatly increased.

ISSN: 0010-8189

(C) CONVERTER 2020 
Generally, a better effect can be achieved when $l$ value is between 3 and 7 . The value of parameter $\mathrm{H}$ is more subjective. In general, when $H$ is smaller (less than 0.2 , for example), the image is sharper, while when $H$ is larger, the image edge is softer. Its specific value should be determined according to the actual demand.

\subsection{Mark non-fractured areas}

After the above two steps, the gray value of the fracture area and the gray value of the core skeleton have been roughly separated, which can be preliminarily marked with threshold value. First of all, we need a preliminary estimate of the proportion of the fracture region in the whole image $\alpha$. Then, a gray threshold $p$ is obtained according to this ratio. The gray value of the pixel that is obviously not a crack in the image is set to 127 , and the pixel value of the remaining points is linearly stretched to the interval $[0,126]$. Algorithm 3 is:

Algorithm 3
Step 1: Estimate the proportion of the crack region $\alpha$ in the whole image. The gray value of pixels in the
image is sorted from small to large, and the corresponding pixel gray value $p$ of $\alpha$ is taken out.
Step 2: Reassignment of pixels in an image. When the original pixel gray value $x<p$, the grey value set
to $x:=127 x / p$; Otherwise set to $x:=127$.

After preliminary threshold marking, the pixels in the image with a gray value of $[0,126]$ basically belong to the crack region, but there is still noise in the image, in addition, the edge of the crack needs to be further determined.

\subsection{Quadratic denoising based on graph}

First not consider noise, basic can correspond to the gray value of the crack area $[0,120],[0,123]$ and $[0,126]$ of the three types pixels, minimum $[0,120]$ of the corresponding crack area, center $[0,123],[0,126]$ the corresponding crack area is the largest, concrete cracks which collection as a need to compare the original image, according to the crack edge pattern to carry on the subjective judgment. After determining the pixel set $[0, P]$ corresponding to the crack, we used the connected area search method ${ }^{[15]}$ to remove the remaining noise. Algorithm 4 is as follows:

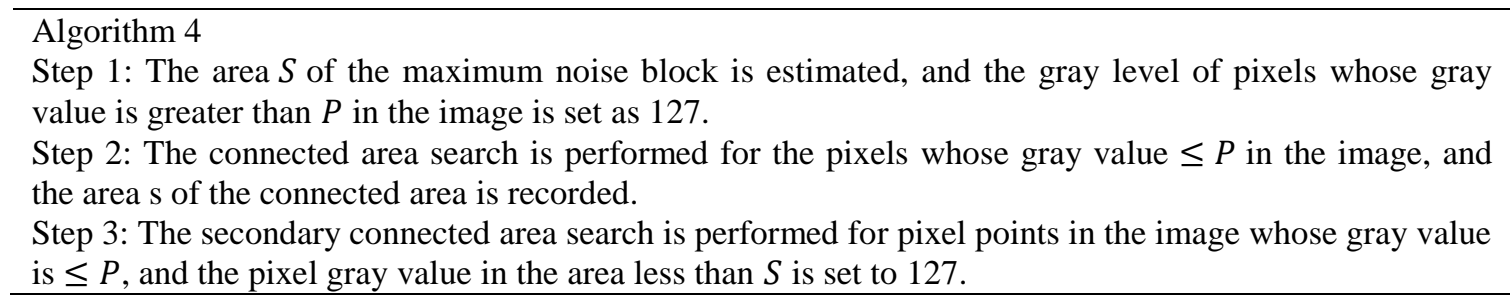

After this de-noising, the noise not connected with the crack is safely removed, and only the crack with pixel gray level of $[0, \mathrm{P}]$ and the background with gray level of 127 are in the image. At this point, the fracture identification of two-dimensional core section is completed.

\section{Case and Result Analysis}

We test the algorithm with a two-dimensional cross-section of the core column shown in Figure 1. In order to analyze the effect of this algorithm, the crack in Figure. 1 is identified by the direct threshold segmentation method. The black region in Figure. 2 is the fractured region identified by the direct threshold method. In Figure. 2a, the threshold range is $[48,90]$. The lower limit of threshold 48 and the upper limit 90 are close to the optimal value. It can be seen from Figure $2 b$ that when the upper threshold value is 100 , the noise in the center of the disk almost covers the crack area, and the recognition fails. As you can see, even with the optimal threshold, the effect of Figure. 2a is quite flawed. First, only the cracks in the central region of the disk were identified, and the edges were completely lost. Secondly, the noise in the central region of the disk is very large, and the noise pixels adhere to the identified fracture region, which is difficult to be removed by post-processing method.

ISSN: 0010-8189

(C) CONVERTER 2020 


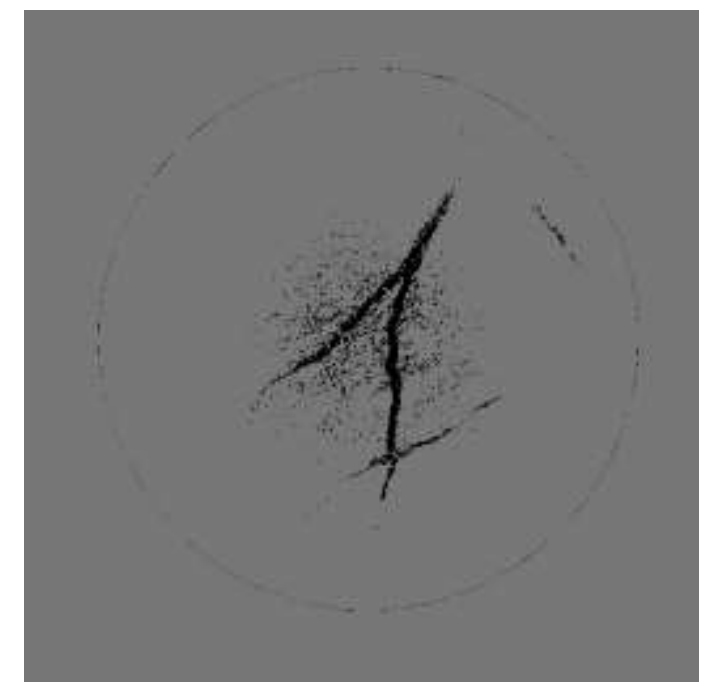

Figure 2a: The region with a threshold of [48,90] in Figure 1

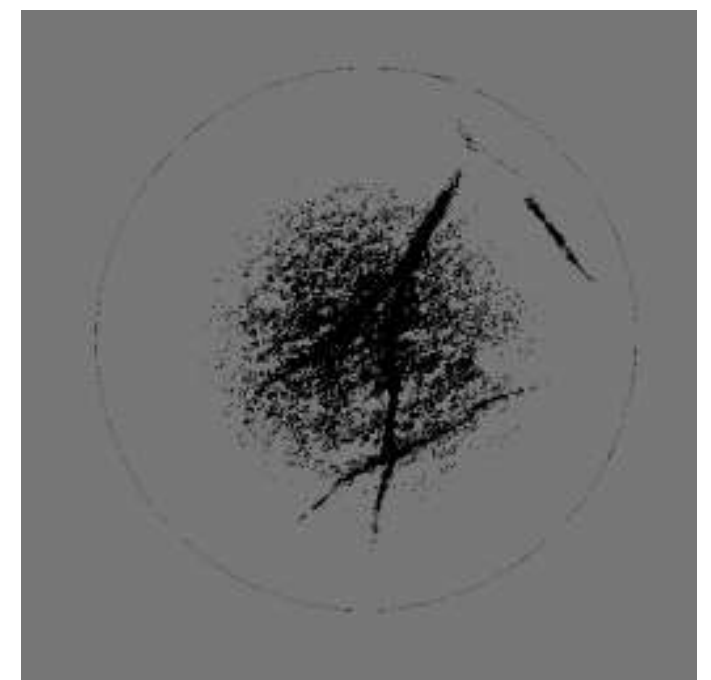

Figure 2b: The region with a threshold of $[48,100]$ in Figure 1

The following algorithm is used for identification. First, adjust the background color difference. Figure 3 compares the difference between the image with the background color difference removed (Figure $3 \mathrm{a}$ ) and the original image (Figure 3b). It can be clearly seen that the whitened edges of the original image have been corrected and the background color of the entire disk is very uniform, while the sharpness of the visible crack areas has not decreased.

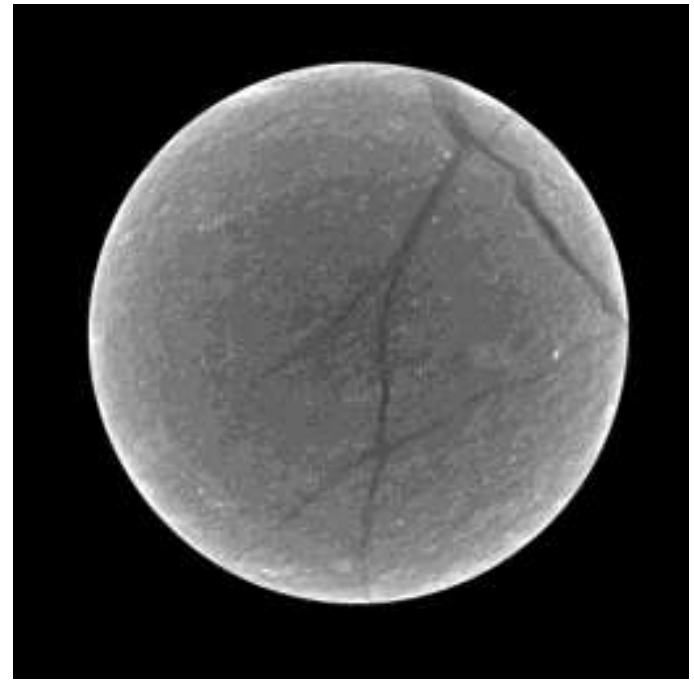

Figure 3: As shown in figure 1

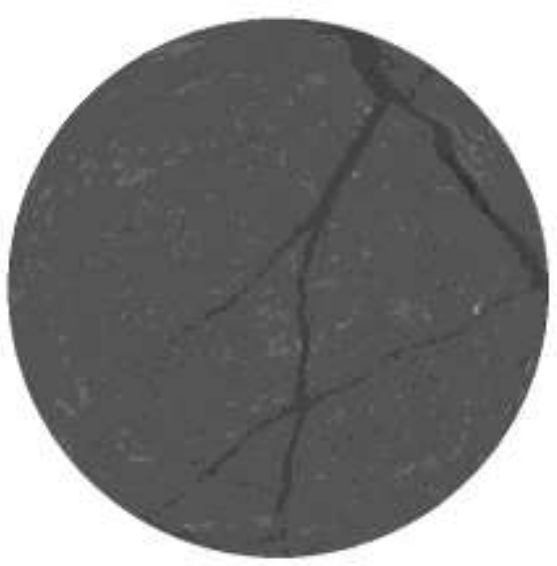

Figure 3b: Eliminate the background color difference and set the air to white

In the second step, non-local mean filtering is applied to Figure 3b. For the three denoising parameters, we first take the window length $\mathrm{L}=7$ for the fixed Gaussian low-pass filter and the similar window length $\mathrm{L}=3$. Then take two different quasi-standard deviations, $\mathrm{h}=0.1$ and $\mathrm{H}=0.2$ for comparison. Figure. 4 shows the results of non-local mean denoising under two groups of different parameters. 

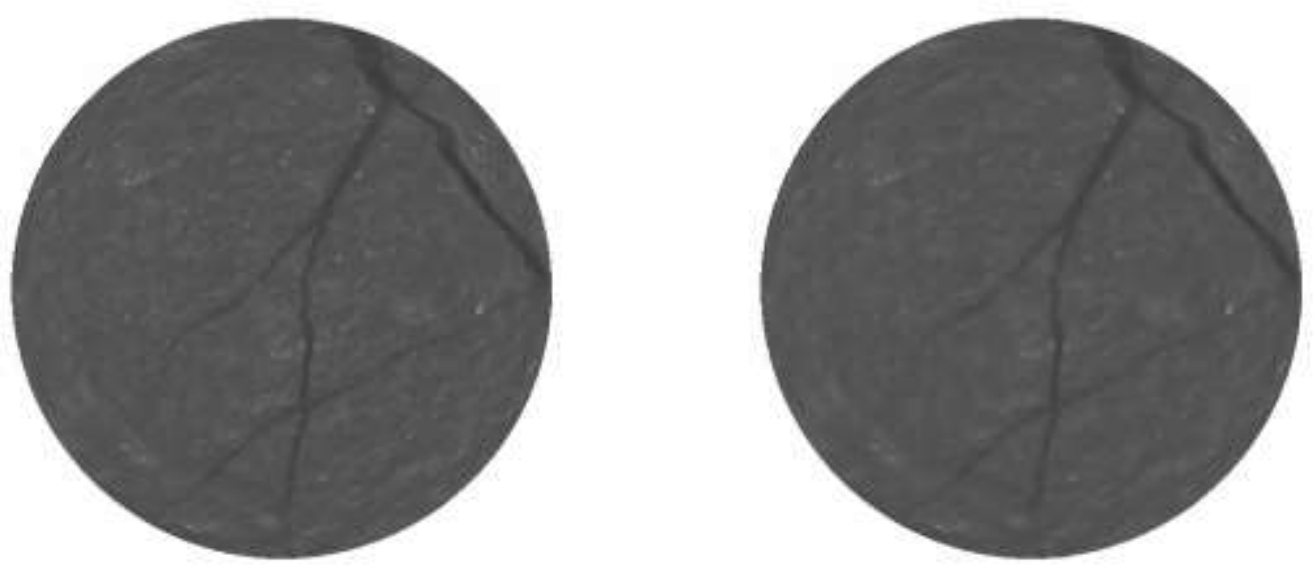

Figure 4a: Non-local mean filtering $h=0.1 \quad$ Figure $4 b$ : Non-local mean filtering $h=0.2$

Since the denoising effect is difficult to be directly discerned by the naked eye, we made partial magnification of the fissure bifurcation in Figure. $3 \mathrm{~b}$ and Figure.4. It can be seen from Figure. $5 \mathrm{~b}$ that when $\mathrm{H}=0.1$, the granular noise in Figure. $3 \mathrm{~b}$ has been basically eliminated on the premise that the crack edge is kept. Increase the value of $\mathrm{H}$, when $\mathrm{H}=0.2$, the noise is less, the image is smoother, and the edge of the crack is more blurred. In general, $\mathrm{h}=0.1$ is a more appropriate parameter.

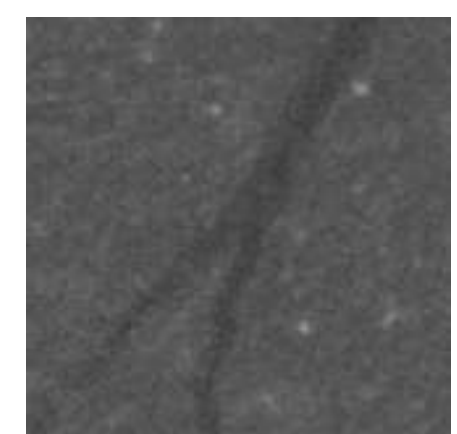

Figure 5a: partial magnification of Figure $3 b$

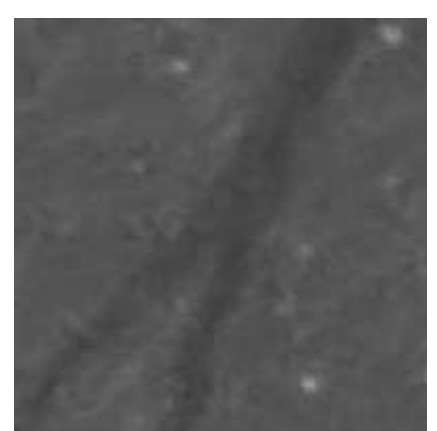

Figure 5b: A partial magnification of Figure $4 a$

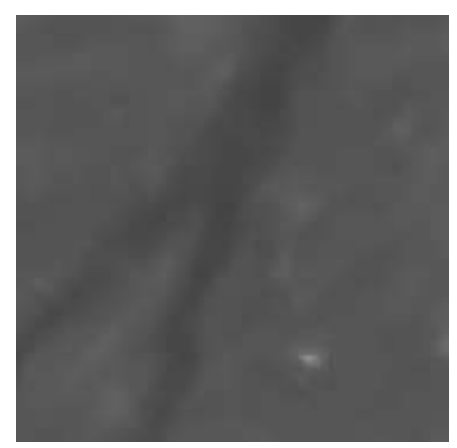

Figure 5c: A partial magnification of Figure $4 b$

The third step is to mark the non-fracture zone and stretch the fracture zone linearly. Here, we estimate that the area of the fracture region accounts for about $=0.05$ of the total image. Figure 6 shows the marked and stretched results of Figure 4. When this step is completed, the dark gray fissure area has been separated from the background. Figure. $6 \mathrm{a}$ and figure.6b, it is hard to see the difference by the naked eye, but in fact they both have noise close to the background color. 


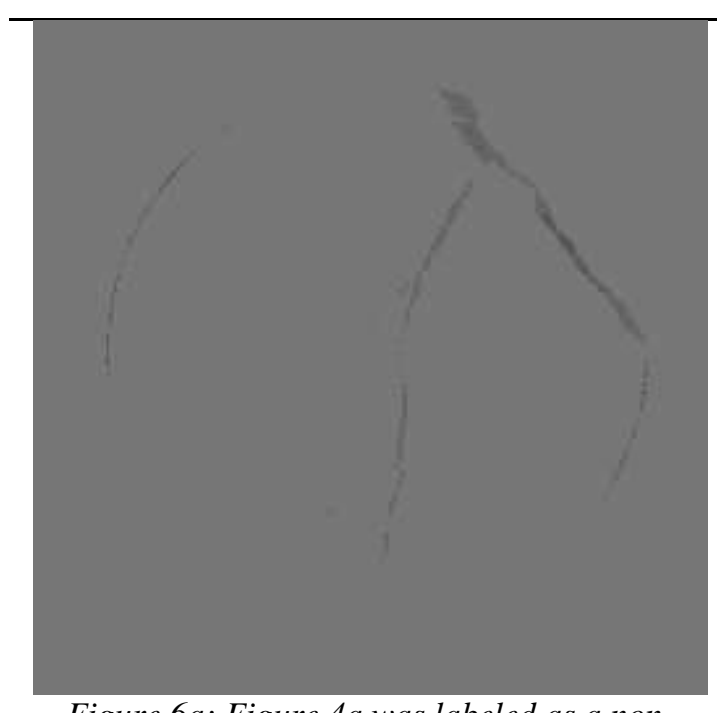

Figure 6a: Figure 4a was labeled as a nonfractured zone

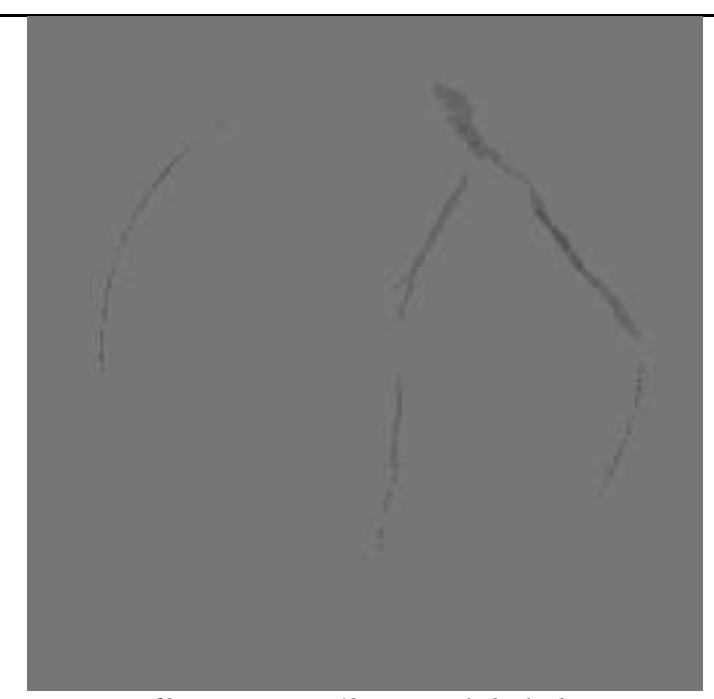

Figure 6b: Figure $4 b$ was labeled as a nonfractured zone

In the fourth step, the graph method is used to remove the noise. By comparing the three types of pixel points in Figure. 6, whose gray values are [0,120], [0,123] and [0,126], Figure. 7 binarized Figure. 6 with pixel value 126 as the threshold value. We found that the pixels with a gray value of $[0,126]$ can not only mark the vast majority of the fracture areas, but also the remaining noise is basically disconnected from the fracture areas. Therefore, we can take $\mathrm{P}=126$ and use the connected region search method to remove the remaining noise.

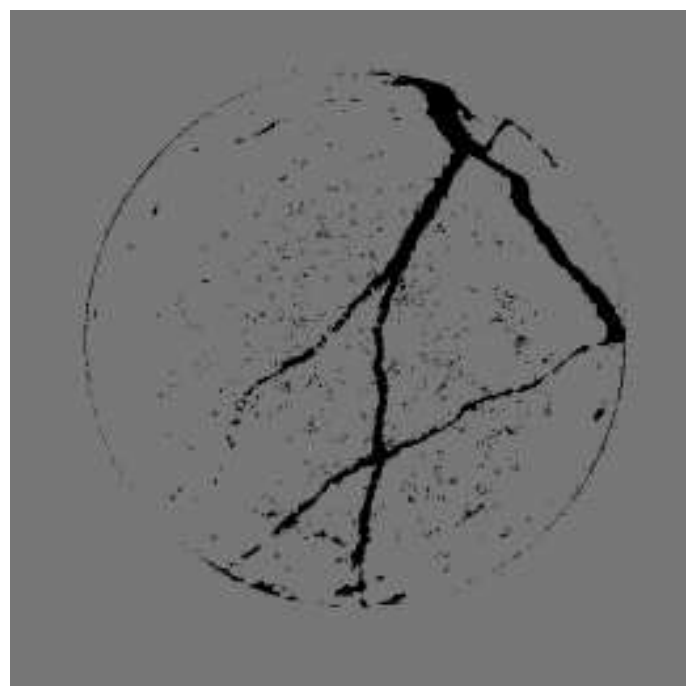

Figure 7a: Cracks and noise in Binarization Figure $6 a$

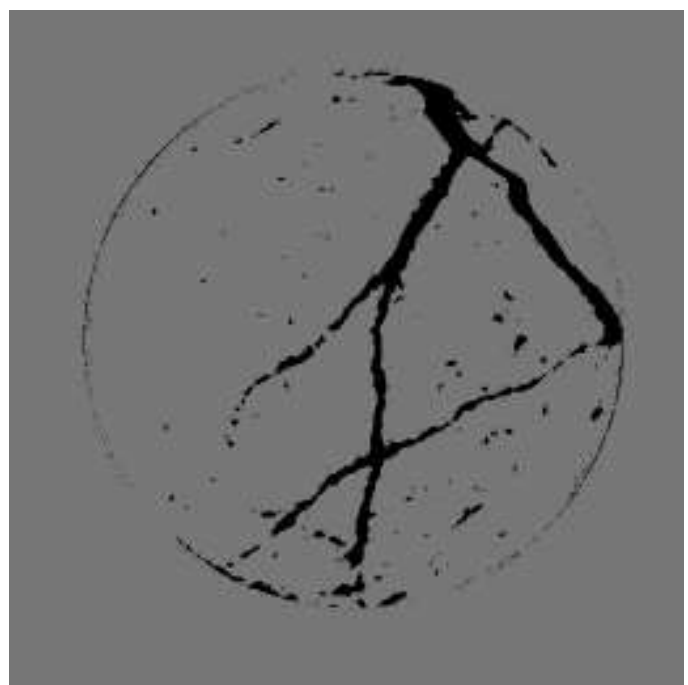

Figure 7b: Cracks and noise in Binarization Figure $6 a$

Figure. 8a and Figure. $8 \mathrm{~b}$ give the final result of denoising the connected region in Figure.6a and Figure. $6 \mathrm{~b}$ respectively. As the noise particles in FIG. 7a are small, the threshold value of denoising area is $\mathrm{S}=48$. In Figure. $7 \mathrm{~b}$, the noise particles are relatively large, and the threshold of denoising area is $S=128$. As can be seen in Figure.8b, in order to remove the noise of large particles, part of the fracture endings that are not connected with the main fracture region are lost. But even so, the recognition effect of Figure 8 is far better than that of the direct threshold partition in Figure. 2a. 


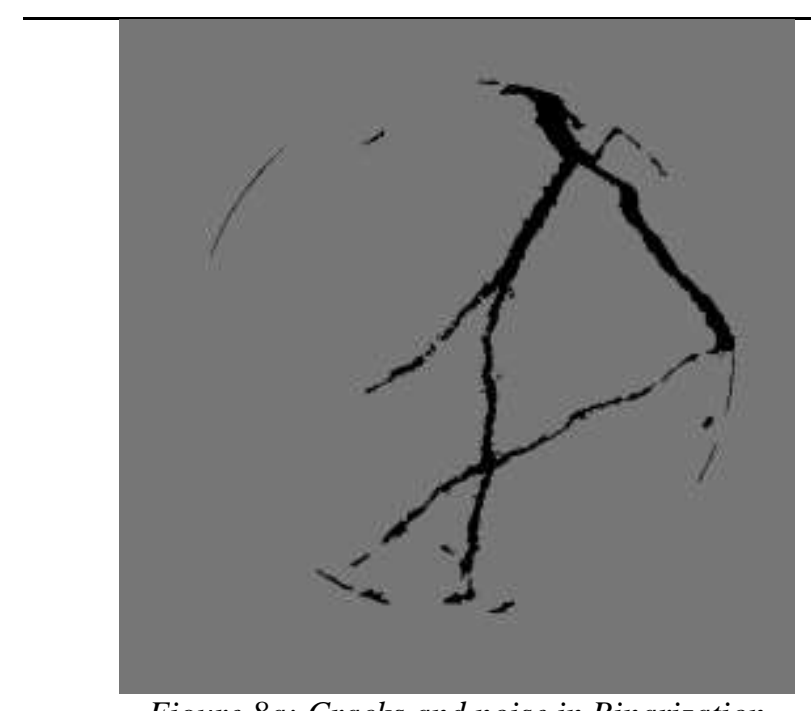

Figure 8a: Cracks and noise in Binarization Figure $6 a$

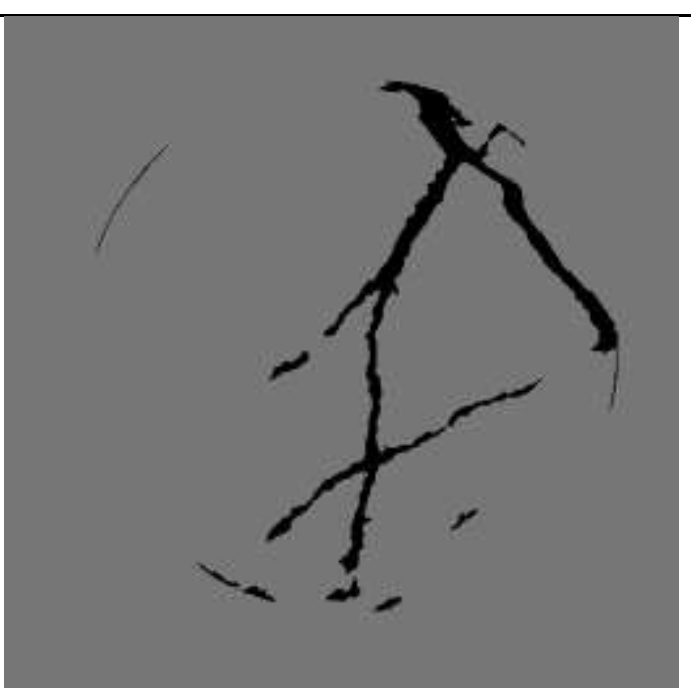

Figure 8b: Cracks and noise in Binarization Figure $6 a$

\section{Conclusion}

Taking core fracture identification as an example, this paper studies the axial $2 \mathrm{~d}$ cross-section of cylindrical core with fractures from the perspective of image processing, analyzes and proposes a general technique for fracture identification in the cross-section. This technique is composed of four steps, including fine tuning background color difference, filtering denoising, marking non-fractured region, and secondary denoising by graphical method. It contains four algorithms. Except algorithm 2, which is a classical algorithm, the rest are autonomous algorithms. It can be seen from the case analysis that, with appropriate parameters, this technique can identify the crack area with almost no noise and accurate boundary. The application of this technique is not only limited to fracture identification of core, but also has a wide application prospect in other fields.

\section{References}

[1] J. B. Shi, J. Malik, "Normalized cuts and image segmentation," IEEE Transactions on Pattern Analysis and Machine Intelligence, vol. 22, no. 8, pp. 888-905, 2000.

[2] D. G. Lowe, "Distinctive image features from scale-invariant keypoints," International Journal of Computer Vision, vol. 60, no. 2, pp. 91-110, 2004.

[3] R. Wang, C. Li, J. Wang, et al., "Threshold segmentation algorithm for automatic extraction of cerebral vessels from brain magnetic resonance angiography images," Journal of Neuroscience Methods, vol. 241, pp. 30-36, 2015.

[4] Y. I. Ohta, T. Kanade, S. Toshiyuki, “Color information for region segmentation,” Computer Graphics and Image Processing, vol. 13, no. 3, pp. 222-241, 1980.

[5] M. Mueller, K. Segl, H. Kaufmann, "Edge and region based segmentation technique for the extraction of large, man-made objects in high-resolution satellite imagery," Pattern Recognition, vol. 37, no. 8, pp. 1619-1628, 2004.

[6] S. Chakraborty, N. K. Goyal, "Irredundant subset cut enumeration for reliability evaluation of flow networks," IEEE Transactions on Reliability, vol. 64, no. 4, pp. 1194-1202, 2015.

[7] S. H. Lee, J. Y. Choi, et al., "Local color vector binary patterns from multichannel face images for face recognition,” IEEE Transactions on Image Processing, vol. 2, no. 4, pp. 2347-2353, 2012.

[8] G. Javier, M. Sebastien, et al., "Image quality assessment for fake biometric detection: application to iris, fingerprint, and face recognition," IEEE Transactions on Image Processing, vol. 23, no. 2, pp. 710 724, 2014.

[9] P. D. Shi, S.Y. Yuan, et al., "Fracture Identification in a tight sandstone reservoir: a seismic anisotropy and automatic multisensitive attribute fusion framework," IEEE Geoscience and Remote Sensing

ISSN: 0010-8189 
Letters, vol. 15, no. 10, pp. 1525-1529, 2018.

[10] N. Shamul, L. Joskowicz, "Radon space dose optimization in repeat ct scanning," IEEE Transactions on Medical Imaging, vol. 36, no. 12, pp. 2436-2448, 2017.

[11] Batard, T. Berthier, et al., "Spinor fourier transform for image processing," IEEE Journal of Selected Topics in Signal Processing, vol. 7, no. 4, pp. 605-613, 2013.

[12] X. L. Cheng, X. Ye, "Analysis of the checkerboard mode and inf-sup condition," Communications in Numerical Methods in Engineering, vol. 18, no. 6, pp. 451-458, 2010, 18(6): 451-458.

[13] N. Otsu, "A threshold selection method from gray-level histogram," IEEE Trans, 1979, pp. 62-66.

[14] Buades, B. Coll, J. M. Morel, "A non-local algorithm for image denoising," Computer Vision and Pattern Recognition, CVPR 2005, IEEE Computer Society Conference on. IEEE, 2005.

[15] M. Jablonski, "Handel-C implementation of classical component labelling algorithm," Digital System Design, Euromicro Symposium. 2004. 